\title{
Three Dimensional (3D) Head Data Classification Based on a Local Shape Feature Description
}

\author{
X.H. ZHENG ${ }^{a, b}$, J.W. NIU ${ }^{c}$, S.T. DING ${ }^{b}$, Q.X. ZHOU ${ }^{a}$ \\ ${ }^{a}$ School of Biological Science and Medical Engineering, Beihang University, China \\ ${ }^{b}$ Research Institute of Chemical Defense, Beijing, China \\ ${ }^{c}$ Department of Logistics Engineering, University of Science and Technology Beijing, Beijing, China
}

\begin{abstract}
Three Dimensional (3D) anthropometric data can be obtained more easily than decades before, but to process $3 \mathrm{D}$ data is most challenging and $3 \mathrm{D}$ shape classification based on local feature is one of the challenges. In this paper, a local shape description based on spin-image, combined with clustering method for 3D head data analysis, is proposed. Numerous methods have been proposed to extract local features of landmarks on 3D head data. But the method with high efficiency, good robustness and strong adaptability is not found out yet. An approach using spin image was adopted in this study to describe the 3D head landmark features. For each head, eight landmarks were selected and their responding spin-images were calculated. In this way, a head can be transformed from point cloud to a vector formed by spin images. K-means clustering algorithm was performed on the transformed data to classify the samples into seven groups. Totally one hundred 3D head data of young male Chinese have been analyzed to illustrate the applicability of the method. Classifying a population of 3D data using this method may provide a promising way to improve product design for fitting comfort. Whether the method in this paper is suitable for 3D data classification of other body segment deserves much more investigation
\end{abstract}

Keywords: Three dimensional (3D), local feature description, spin image, clustering

\section{Introduction}

The application of the 3D anthropometric data was very widespread. In recent years, several large 3D anthropometric surveys have been conducted, such as CAESAR (Civilian American and European Surface Anthropometry Resource) (Robinette et al 2002), SizeUK (Bougourd et al. 2004), SizeUSA (http://www.tc2.com), SizeChina (www.sizechina.com) and the work at the Research Institute of Human Engineering for Quality Life in Japan (http://www.hql.or.jp), etc. Three dimensional data was important to product design in the industry of garment, tooling, and furniture, etc. Three dimensional anthropometric surveys aroused the researchers' interest and a number of researchers have devoted themselves to the 3D data processing research. The technology of the 3D anthropomety raised a number of research topics, among which 3D data classification was the most typical one. An effective way to design fitting products is to analyze human body forms and to classify them into several groups, commonly referred to as "sizing" in anthropometry. A sizing system classifies a specific population into homogeneous groups. The studies of traditional sizing system almost focused on $2 \mathrm{D}$ measurements such as stature and chest girth. There are some drawbacks in traditional sizing methods. The most important is that, in traditional uni-variate or bi-variate sizing methods, only one or two dimensions are referred to. Geometric characteristics and internal structure of human surface are not adequately considered. This may lead to design deficiency on fitting comfort. Some other researchers also tried to combine 2D with 3D data, but the research of 3D anthropometric data classification was very limited. This paper is organized as follows: the theoretical background of spin image will be introduced in chapter 2. The samples and the results of our research will be introduced in chapter 3 and chapter 4 . In chapter 5 , we will summarize our paper and give some discussion. We will conclude our work in chapter 6.

*zheng_nudt@163.com 


\section{Methodology}

Spin image was first proposed by Johnson [Johnson, 1997]. He developed a surface matching representation system. A dense collection of $3 \mathrm{D}$ points, surface normal and spin images associated with each point were taken into consideration in this system.

Each spin image is associated with a 3D point. Namely, the point and spin image always appear in pairs. The spin image describes the feature of finite area around the point and the point is used to created spin image as an oriented point. The nature of spin image is the projection of the relative position of 3D points to $2 \mathrm{D}$ space. The spin image of one oriented point can be generated as follows.

\subsection{Spin image definition}

The fundamental components of spin map are oriented point, surface normal and tangent plane associated with the oriented point. As shown in Fig. 1, $p$ is the oriented point, and then its surface normal $n$, tangent plane $P$ and point $p$ compose its spin map. The coordinates of a 3D point $x$ in the spin map is $(\alpha, \beta)$, where $\alpha$ is the perpendicular distance to the surface normal $n$, and $\beta$ is the perpendicular distance to the plane $P$. The coordinates can be computed as follows:

$$
\begin{aligned}
& \alpha=\sqrt{\|x-p\|^{2}-(n \cdot(x-p))^{2}} \\
& \beta=n \cdot(x-p)
\end{aligned}
$$

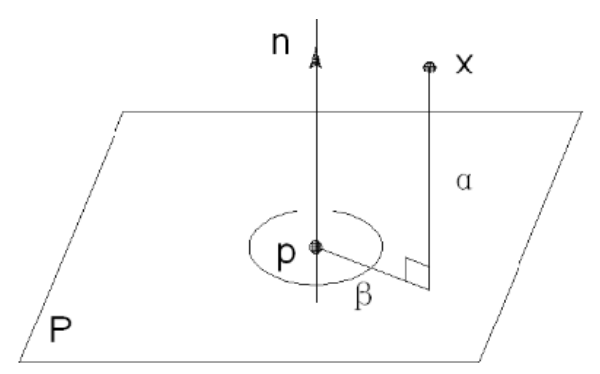

Fig. 1. Spin definition of an oriented point $p$

\subsection{Spin -image generation}

The spin image is a projection of $3 \mathrm{D}$ points to $2 \mathrm{D}$ spin map. It describes the relative position of vertices on a surface with respect to a particular point on that same surface. Each oriented point on the surface of a model has a unique spin map associated with it. When the spin map is applied to all the vertices of a surface mesh, a set of 2D points is created, and we call it spin image. To create a spin image, it can be done as follows:

First, an oriented point is identified from a vertex of the surface mesh. Then, for each vertex $x$ on the surface, the spin map coordinates with respect to the oriented point is computed. If $x$ meets some criteria based on the distance from the oriented point and the angle between oriented point and the surface normal of $x$, the index of bin which point $\mathrm{x}$ is projected in is determined. At last, to update the $2 \mathrm{D}$ array by increasing the surrounding bins in the array.

\subsection{Spin Image parameters}

The spin image has four parameters: bin size $b$, image width $W$, support distance $D s$ and support angle As.

Bin size is a very important parameter because it determines the storage size of the spin image and a suitable bin size may reduce the influence of individual point positions. It also has an effect on the descriptiveness of the spin-images. Image width is the number of rows or columns in a square spin image. Support distance is the result of image width multiplies the bin size. It determines the amount of space swept out by a spin image. Image width or support distance controls the amount of global information in a spin image. For a fixed bin size, decreasing image width will decrease the descriptiveness of a spin image because the amount of global shape included in the image will be reduced. Support angle is the maximum angle between the direction of the oriented point the basis of a 
spin image and the surface normal of points that are allowed to contribute to the spin image. Support angle is used to limit the effect of self occlusion and clutter during spin image matching. Decreasing support angle also has the effect of decreasing the descriptiveness of spin image. Generally, support angle is set between $60^{\circ}$ and $90^{\circ}$

\subsection{K-means clustering}

Cluster analysis was used as an un-supervised data analysis tool for classification. It's the procedures that attempt to find natural partitions of patterns. It concerns how to group a set of objects based on their similarity of attributes. According to clustering mechanisms, clustering methods can be categorized as partitioning method, hierarchical method, density-based method, grid-based method, and model-base method. K-means clustering, known as a widely-used partitioning method, was adopted in this study. In order for the ease of comparison between the current helmet sizing system in China and the proposed method in this paper, seven was selected as the number of clusters $(K)$ for the k-means clustering.

\section{Samples}

In this study, the 3D head data of young male Chinese soldiers (aged from 19 to 23) were collected by a Chinese institute in 2002 [Chen, et al., 2002] The scanning was performed from the top to the neck with slice interval of $5 \mathrm{~mm}$. The data we received are row points of outer surface in each slice and head dimensions. The sample size used for the case study was 100 . The point number of one head sample is about 23,220 . Under the CAD software Unigraphics, noise data were removed manually by visual check [Niu, et al., 2009].

\section{Results}

\subsection{Spin Image results}

Eight facial landmarks were identified manually using the package developed by our colleague under Unigraphics CAD software. The definitions of the eight facial landmarks are based on ISO7250. Then their spin images were generated.

Spin image extracts local features of landmarks and then restores the feature information in a matrix. As shown in Fig. 2, it is a $15 \times 15$ matrix, the first number 15 is the so called image width along $\alpha$ dimension, and the second 15 is the image width in $\beta$ dimension. Red cell 9 in bin $(8,8)$ represents the number of 3D points whose $\alpha$ is between $7 \times b$ and $8 \times b$ and $\beta$ is between $7 \times b$ and $8 \times b$, where $b$ denotes bin size whose value equals $2 \mathrm{~mm}$. If we arrange the spin image matrix values of a landmark into a vector row by row, then the local shape feature of this landmark can be represented in the vector form. Specifically, for example, the $15 \times 15$ spin image matrix of the facial landmark as shown in Fig. 2 , will be transferred into a $1 \times 225$ row vector. Considering totally there are eight landmarks selected from each head as representatives, we transform each head from point cloud into a $8 \times 225$ vector.

Spin image is insensitive to pose and lightning. Our preliminary results show that when the pose of the head changed, the spin image stayed constant. Fig. 3 shows the spin image of a facial landmark. 


\begin{tabular}{|l|l|l|l|l|l|l|l|l|l|l|l|l|l|l|}
\hline 1 & 1 & 1 & 1 & 1 & 1 & 1 & 4 & 1 & 1 & 1 & 1 & 1 & 1 & 1 \\
\hline 1 & 1 & 1 & 1 & 1 & 1 & 1 & 9 & 1 & 1 & 1 & 1 & 1 & 1 & 1 \\
\hline 1 & 1 & 1 & 1 & 1 & 1 & 2 & 6 & 1 & 1 & 1 & 1 & 1 & 1 & 1 \\
\hline 1 & 1 & 1 & 1 & 1 & 1 & 2 & 15 & 4 & 1 & 1 & 1 & 1 & 1 & 1 \\
\hline 1 & 1 & 1 & 1 & 1 & 2 & 5 & 11 & 5 & 1 & 1 & 1 & 1 & 1 & 1 \\
\hline 1 & 1 & 1 & 1 & 1 & 3 & 7 & 8 & 7 & 1 & 1 & 1 & 1 & 1 & 1 \\
\hline 1 & 1 & 1 & 1 & 1 & 5 & 6 & 12 & 10 & 3 & 1 & 1 & 1 & 1 & 1 \\
\hline 1 & 1 & 1 & 1 & 1 & 5 & 11 & 9 & 4 & 5 & 1 & 1 & 1 & 1 & 1 \\
\hline 1 & 1 & 1 & 1 & 1 & 6 & 14 & 10 & 9 & 6 & 3 & 1 & 1 & 1 & 1 \\
\hline 1 & 1 & 1 & 1 & 1 & 6 & 14 & 7 & 9 & 7 & 4 & 1 & 1 & 1 & 1 \\
\hline 1 & 1 & 1 & 1 & 4 & 4 & 15 & 8 & 4 & 8 & 4 & 1 & 1 & 1 & 1 \\
\hline 1 & 1 & 1 & 1 & 5 & 4 & 18 & 12 & 7 & 6 & 7 & 6 & 1 & 1 & 1 \\
\hline 1 & 1 & 1 & 1 & 7 & 7 & 13 & 10 & 10 & 4 & 2 & 5 & 6 & 1 & 1 \\
\hline 1 & 1 & 1 & 2 & 9 & 7 & 7 & 10 & 8 & 4 & 3 & 6 & 4 & 1 & 1 \\
\hline 1 & 1 & 1 & 8 & 9 & 7 & 7 & 13 & 6 & 3 & 3 & 5 & 7 & 8 & 2 \\
\hline
\end{tabular}

Fig. 2. Spin image matrix of a facial landmark

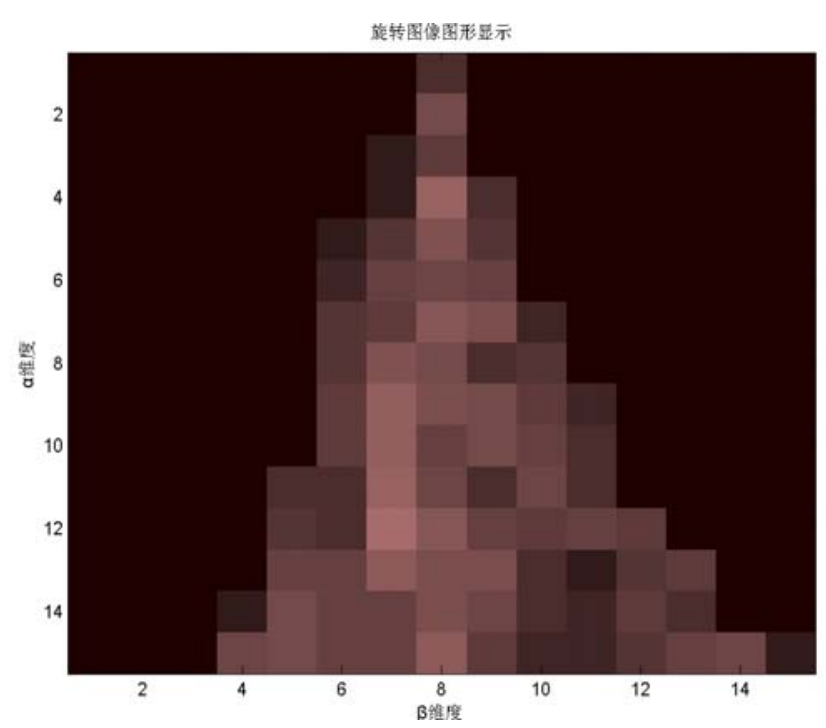

Fig. 3. Spin image of a facial landmark

\subsection{K-means clustering results}

As a case study, one hundred head samples were used in this paper. It can be seen, there are only 6,2 and 5 samples falling into cluster 1,3 and 7 . This is relative quite small compared with the total sample number of 100 . This can be explained that four groups or clusters may be optimal from the point of cost. That is to say, cluster 2, 4, 5 and 6 had better remained. Distances between final cluster centers are as shown in Table 2. For instance, the distance from center of cluster 3 to the centers of cluster 2, 4, 5, 6 and are $87.6,116.6,127.6,100.7$ and 139.9 , respectivelly. 
Table 1 Number of cases in each cluster

$\begin{array}{cc}\begin{array}{c}\text { Cluster } \\ \text { ID }\end{array} & \text { Number in } \\ \text { each cluster } \\ 1 & 6 \\ 2 & 11 \\ 3 & 2 \\ 4 & 26 \\ 5 & 33 \\ 6 & 17 \\ 7 & 5 \\ \text { Total } & 100\end{array}$

Table 2 Distances between final cluster centers

\begin{tabular}{ccccccc}
\hline Cluster & 2 & 3 & 4 & 5 & 6 & 7 \\
\hline 1 & 87.6 & 136.7 & 84.9 & 59.8 & 66.8 & 66.6 \\
2 & & 133.1 & 45.3 & 63.5 & 64.0 & 94.4 \\
3 & & & 116.6 & 127.6 & 100.7 & 139.9 \\
4 & & & & 48.9 & 51.8 & 91.0 \\
5 & & & & & 52.9 & 65.6 \\
6 & & & & & & 79.4 \\
\hline
\end{tabular}

\section{Discussion}

Three dimensional data classification should make full use of the local features on the data surface. To extract local feature, plenty of methods have been proposed. Contrasted with other methods, spin image is simple to compute and scalable for local feature representation. But so far, the spin image method has not attracted extensive attention. The generation parameters can affect the descriptiveness of the spin image. Bin size can decrease the influence of individual point position.

Spin image only derives the local feature of some landmarks. It's recommended to utilize some global feature(s) based on statistics. But the weight appointment on the local and global feature is not a easy task. This must be done through lots of trials and experiments.

Though data mining technique such as k-means clustering can disclose some interesting and meanningful results, domain expertise must be paid enough attention. Three dimensional data classification probably affects product fitting design or workplace comfort improvement, so it should not be based only on mathmatic or statistics.

\section{Conclusion}

In the last decades, much progress has been made for 3D anthropometric data classification. There is not a final conclusion yet to present logical criteria for choosing the optimal method. We proposed a 3D anthropometric data classification method, combined with spin image and k-means clustering. The clustering resuld should be compared with traditional method in future. This work can bridge the gap between 3D data analysis and product fitting design.

\section{Acknowledgment}

The study is supported by the National Natural Science Foundation of China (No.51005016). 


\section{References}

[1] Robinette, K. M., Blackwell, S., Daanen, H., et at., CAESAR, Final Report, Volume I: Summary, AFRL HE WP TR 2002 0169. United States Air Force Research Lab., Human Effectiveness Directorate, Crew System Interface Division, Dayton, Ohio, 2002.

[2] Johnson, A. E., Spin-images: a representation for 3-D surface matching, Ph.D. dissertation, Univ. Carnegie Mellon, Pittsburgh, Pennsylvania, USA, 1997.

[3] Chen, X., Shi, M. W., et al., The standard head for sizing military helmets based on computerized tomography and the headform sizing algorithm (in Chinese), Acta Armamentarii, vol. 23, no. 4, pp. 476-480, 2002.

[4] Niu, J. W., Li, Z. Z., and Salvendy, G., Multi-resolution shape description and clustering of three-dimensional head data. Ergonomics, vol. 52, pp. 240-264, 2009.

[5] International Standard, International Organization for Standardization. 1996-07-15. Basic Human Body Measurements for Technological Design ISO7250. 